$63^{\text {ème }}$ Congrès de la SFCO, 02028 (2015)

DOI:10.1051/sfco/20156302028

(C) Owned by the authors, published by EDP Sciences, 2015

\title{
COMMUNICATION
}

\section{Le myxome odontogène : à propos d'un cas}

\author{
Feugueur G*, Adam L*, Vierne C**, Chemli $\mathrm{H}^{* *}$, Bémer J* \\ * Service odontologie du Groupe Hospitalier du Havre \\ ** Service stomatologie et CMF du Groupe Hospitalier du Havre
}

Le myxome odontogène est une tumeur bénigne qui se développe à partir des tissus mésenchymateux odontogènes, et se retrouve de manière exclusive au niveau des maxillaires (McLure 1977). La prévalence du myxome odontogène est de $0,04 \%$ à 3,7 \% des tumeurs osseuses (Halfpenny 2000) et représente 3,3 \% à 15,7 \% des tumeurs odontogènes chez l'adulte (Kansy 2012). Cette tumeur se développe préférentiellement chez le jeune de 20 à 30 ans (Piette 1977) de manière lente et souvent asymptomatique. Cependant, elle est localement agressive avec envahissement des tissus mous comme l'illustre parfaitement ce cas clinique.

Il s’agit d’une patiente de 16 ans adressée par son médecin généraliste aux urgences pédiatriques pour suspicion d'un abcès dentaire mandibulaire postérieur droit. La patiente a un contexte familial difficile et un suivi médical irrégulier. Elle sera cependant traitée à multiples reprises par antibiothérapie pour cet abcès supposé. La patiente rapporte une évolution douloureuse depuis quatre mois de cette masse intra buccale devenant gênante à la mastication.

En exo-buccal, la patiente présente aucune adénopathie, mais une légère hypoesthésie labiomentonnière et une limitation de l'ouverture buccale.

L'examen endo-buccal montre une hygiène déficiente, des caries multiples dont une carie profonde sur 47 qui est nécrosée. On note la présence d'une masse gingivale en distal de 47 d'aspect fibreux, mou à la palpation et saignant car en occlusion avec l'arcade supérieur. La tuméfaction est sensible à la palpation.

Des examens radiologiques complémentaires ont été réalisés (OPT, scanner). Le scanner rapporte une lésion homogène, aux contours réguliers, bilobée. La 48 est refoulée par la lésion vers le processus coronaire de la mandibule. Les corticales osseuses vestibulaires et linguales sont soufflées.

La biopsie osseuse réalisée sous anesthésie générale a été envoyée pour analyse anatomo-pathologique de la lésion. Le compte rendu indique un prélèvement faiblement cellulaire, constitué de substance fondamentale claire, mucoïde. Les cellules sont effilées, à noyau dense et activité mitotique faible.

Les résultats évoquent un myxome odontogène à point de départ de la dent 48.

La thérapeutique sera la chirurgie d'exérèse et avulsion de la 48. Un curetage appuyé des berges osseuses avec conservation du nerf lingual et nerf alvéolaire inférieur est effectué. En effet les récidives sont d'en moyenne 25 \% (YokoKawase 2014) à 31,2 \% (Gassab 2007) à la mandibule. Les récurrences maxillaires sont beaucoup plus fréquentes et demandent un curetage plus appuyé de la lésion initiale (Slootweg 1986).

This is an Open Access article distributed under the terms of the Creative Commons Attribution License 4.0, which permits unrestricted use, distribution, and reproduction in any medium, provided the original work is properly cited. 
La prise en charge tardive de la lésion de cette patiente, de part l'errance diagnostique, nous pousse à constater la nécessité de former les professionnels de santé à la détection des tumeurs bénignes des maxillaires et d'améliorer le diagnostic différentiel avec les infections et kystes d'origine dentaire.

Nom et adresse du conférencier

\section{Guillaume FEUGUEUR}

Service odontologie du groupe hospitalier du Havre

55 bis rue Gustave Flaubert

76600 le Havre (France)

guillaume.feugueur@hotmail.fr 